\title{
Topological Properties of Para-Line Graph of Some Convex Polytopes Using Neighborhood M-Polynomial
}

\author{
Sourav Mondal 1(i), Nilanjan De ${ }^{2 * * i D}$, Muhammad Kamran Siddiqui ${ }^{3}$ (D), Anita Pal ${ }^{1}$ (D) \\ 1 National Institute of Technology Durgapur, India; souravmath94@gmail.com (S.M.); anita.buie@ gmail.com (A.P.); \\ Calcutta Institute of Engineering and Management, Kolkata, India; de.nilanjan@ rediffmail.com (N.D.); \\ COMSATS University Islamabad, Lahore Campus, Pakistan; kamransiddiqui75@gmail.com (M.K.S.); \\ * Correspondence: de.nilanjan@ rediffmail.com;
}

Scopus Author ID 55385439300

Received: 31.08.2020; Revised: 17.09.2020; Accepted: 19.09.2020; Published: 23.09.2020

\begin{abstract}
The neighborhood M-polynomial is effective in recovering neighborhood degree sum based topological indices that predict different physicochemical properties and biological activities of molecular structures. Topological indices can transform the information found in molecular graphs and networks into numerical characteristics and thus make a major contribution to the study of structureproperty and structure-activity relationships. In this work, the neighborhood M-polynomial of the paraline graph of some convex polytopes is obtained. From the neighborhood M-polynomial, some neighborhood degree-based topological indices are recovered. Applications of the work are described. In addition, a quantitative and graphical comparison is made.
\end{abstract}

Keywords: convex polytopes; line graph; neighborhood M-polynomial; subdivision graph; topological index.

(C) 2020 by the authors. This article is an open-access article distributed under the terms and conditions of the Creative Commons Attribution (CC BY) license (https://creativecommons.org/licenses/by/4.0/).

\section{Introduction}

Apart from certain mathematical sciences, the major area of implementation of graph theory is in chemistry. The great import of graph theory in chemistry has come to be widely recognized in chemical graph theory, where an outstanding area is preserved for the wellknown topological indices. A topological index is a numeric quantity associated with a graph that describes the topology of the graph and is invariant under the graph isomorphism. The topological indices are used for the correlation and prediction of a very broad range of molecular properties. These range from the physical, through the chemical, the thermodynamic, the biochemical, the pharmacological, and the toxicological. The journey of topological indices was initiated by $\mathrm{H}$. Wiener [1] while working on the boiling point for paraffin. Then a large number of topological indices have been developed. Recently researchers have put their attention to design topological indices based on the neighborhood degree of vertex [2-8]. By the neighborhood degree of a node, we mean the totality of degrees of all nodes that are connected to the node. The degree of a vertex is the total number of edges incident to the vertex. The computation of a certain type of topological indices using their usual definitions is laborious. To overcome this approach, many algebraic polynomials [9-11] have been introduced whose differentiation or integration or both obtained at some particular point yield topological indices. For degree-based topological indices, M-polynomial [14-16] is a very effective tool. To make the computation of neighborhood degree-based topological indices easier, present authors introduced neighborhood M-polynomial [17] whose role for 
neighborhood degree-based indices is parallel to the role of the M-polynomial for degree-based indices. The subdivision [18] of a graph $G$, denoted by $S(G)$, is obtained by inserting an additional vertex to each edge of $G$. The line graph [19] of a graph $G$ is another graph $L(G)$ such that each vertex of $L(G)$ correspond to an edge of $\mathrm{G}$ and two vertices of $L(G)$ are adjacent if and only if their corresponding edges share a common endpoint in $G$. By para-line graph [20,21] of a graph $G$, we mean the line graph of subdivision graph $(L(S(G)))$ of $G$. Convex polytopes are basic geometrical objects. The beauty of their theory is now complemented by its significance for many other mathematical topics, from integration theory, algebraic topology, and algebraic geometry to linear and combinatorial optimization [22]. Also, people are paying attention to finding the metric dimension and labeling of convex polytopes [23,24]. The goal of this article is to obtain some neighborhood degree-based indices of the para-line graph of some convex polytopes using the neighborhood M-polynomial approach.

The rest of the present work is constructed as follows: Some basic definitions and formulas that are useful to obtain results are given in section 2 . The materials and methods required to build the results are illustrated in section 3. Section 4 deals with the main computational results that are divided into three subsections for three para-line graphs. The results are compared numerically and graphically in section 5 . We conclude the whole work in section 6 .

\section{Preliminaries}

Throughout this article, we consider a simple connected graph. Let $V(G)$ and $E(G)$ be the vertex set and edge set of a graph $G$ respectively. By $\delta_{u}$ we mean the neighborhood degree of $u \in V(G)$.

The neighborhood M-polynomial [17] of a graph $G$ is defined as,

$$
N M(G ; x, y)=\sum_{i \leq j} m_{(i, j)} x^{i} y^{j} .
$$

Where $m_{(i, j)}$ is the total number of edges $u v \in E(G)$ such that $\left\{\delta_{u}, \delta_{v}\right\}=\{i, j\}$. Weuse $N M(G)$ for $N M(G ; x, y)$ for the rest of the article. The Neighborhood degree-based topological indices defined on the edge set of a graph $G$ can be expressed as

$$
I(G)=\sum_{u v \in E(G)} f\left(\delta_{u}, \delta_{v}\right),
$$

where $f\left(\delta_{u}, \delta_{v}\right)$ is the function of $\delta_{u}, \delta_{v}$ used in the definition of neighborhood degree-based indices. The above result can also be written as,

$$
I(G)=\sum_{i \leq j} m_{(i, j)} f(i, j) .
$$

Now we describe some neighborhood degree-based topological indices.

The third version of the Zagreb index [3] is defined as,

$$
M_{1}^{\prime}(G)=\sum_{u v \in E(G)}\left(\delta_{u}+\delta_{v}\right) .
$$

The neighborhood second Zagreb index [7] is defined as,

$$
M_{2}^{*}(G)=\sum_{u v \in E(G)} \delta_{u} \delta_{v} .
$$

The neighborhood forgotten topological index [7] is defined as,

$$
F_{N}^{*}(G)=\sum_{u v \in E(G)}\left(\delta_{u}{ }^{2}+\delta_{v}{ }^{2}\right) .
$$

The neighborhood second modified Zagreb index [17] is defined as,

$$
M_{2}^{n m}(G)=\sum_{u v \in E(G)} \frac{1}{\delta_{u} \delta_{v}} .
$$

The neighborhood general Randić [17] index is defined as,

$$
N R_{\alpha}(G)=\sum_{u v \in E(G)}\left(\delta_{u} \delta_{v}\right)^{\alpha} .
$$

The third NDe index [8] is defined as, 


$$
N D_{3}(G)=\sum_{u v \in E(G)} \delta_{u} \delta_{v}\left(\delta_{u}+\delta_{v}\right)
$$

The fifth NDe index [8] is defined as,

$$
N D_{5}(G)=\sum_{u v \in E(G)}\left[\frac{\delta_{u}}{\delta_{v}}+\frac{\delta_{v}}{\delta_{u}}\right] .
$$

The Harmonic neighborhood index [17] is defined as,

$$
N H(G)=\sum_{u v \in E(G)} \frac{2}{\delta_{u}+\delta_{v}} .
$$

The neighborhood inverse sum index [17] is given by

$$
N I(G)=\sum_{u v \in E(G)} \frac{\delta_{u} \delta_{v}}{\delta_{u}+\delta_{v}}
$$

The Sanskruti index [2] is defined as,

$$
S(G)=\sum_{u v \in E(G)}\left(\frac{\delta_{u} \delta_{v}}{\delta_{u}+\delta_{v}-2}\right)^{3} .
$$

The relations of some neighborhood degree-based topological indices with the NM-polynomial are shown in Table 1.

Table 1. Derivation of some neighborhood degree-based topological indices.

\begin{tabular}{c|c|c} 
Topological index & $\boldsymbol{f}(\boldsymbol{x}, \boldsymbol{y})$ & Derivation from $\boldsymbol{N} \boldsymbol{M}(\boldsymbol{G})$ \\
\hline$M_{1}^{\prime}$ & $x+y$ & $\left.\left(D_{x}+D_{y}\right)(N M(G))\right|_{x=y=1}$ \\
\hline$M_{2}^{*}$ & $x y$ & $\left.\left(D_{x} D_{y}\right)(N M(G))\right|_{x=y=1}$ \\
\hline$F_{N}^{*}$ & $\frac{x^{2}+y^{2}}{x y}$ & $\left.\left(S_{x} S_{y}\right)(N M(G))\right|_{x=y=1}$ \\
\hline$M_{2}^{n m}$ & $\frac{1}{x y}{ }^{\alpha}$ & $\left.\left(D_{x}{ }^{\alpha} D_{y}{ }^{\alpha}\right)(N M(G))\right|_{x=y=1}$ \\
\hline$N R_{\alpha}$ & $\mathrm{xy}(\mathrm{x}+\mathrm{y})$ & $\left.D_{x} D_{y}\left(D_{x}+D_{y}\right)(N M(G))\right|_{x=y=1}$ \\
\hline$N D_{3}$ & $\frac{x^{2}+y^{2}}{x y}$ & $\left.\left(D_{x} S_{y}+S_{x} D_{y}\right)(N M(G))\right|_{x=y=1}$ \\
\hline$N D_{5}$ & $\frac{x}{x+y}$ & $\left.2 S_{x} J(N M(G))\right|_{x=y=1}$ \\
\hline$N H$ & $\frac{x y}{x+y}$ & $\left.S_{x} J D_{x} D_{y}(N M(G))\right|_{x=y=1}$ \\
\hline$N I$ & $\frac{x y}{(x+y-2)^{3}}$ & $\left.S_{x}{ }^{3} Q_{-2} J D_{x}{ }^{3} D_{y}{ }^{3}(N M(G))\right|_{x=y=1}$
\end{tabular}

Where,

$D_{x}(f(x, y))=x \frac{\partial(f(x, y))}{\partial x}, \quad D_{y}(f(x, y))=y \frac{\partial(f(x, y))}{\partial y}, S_{x}(f(x, y))=\int_{0}^{x} \frac{f(t, y)}{t} d t$,

$S_{y}(f(x, y))=\int_{0}^{y} \frac{f(x, t)}{t} d t, J(f(x, y))=f(x, x), Q_{\alpha}(f(x, y))=x^{\alpha} f(x, y)$.

For some recent works on this field, readers are referred to [25-30].

\section{Materials and Methods}

Our main computational results include NM-polynomial and some neighborhood degree sum based topological indices of the para-line graph of convex polytopes. We utilize combinatorial computation, edge partition method, graph-theoretical tools, analytical techniques, and neighborhood degree sum counting method to derive our results. The surface plotting of NM-polynomial is made by Maple 2015.1. The results are described graphically using Matlab 2017. 


\section{Results and Discussion}

\subsection{Main results.}

In this section, we obtain topological indices for the para-line graph of three types of convex polytopes using NM-polynomial. We consider the following three subsections.

4.1.1. Para-line graph of the convex polytope $\mathrm{R}_{\mathrm{p}}$.

A Cartesian product of a cycle graph $\left(C_{p}\right)$ and a path graph of order $\left(P_{2}\right)$ yields a prism graph. A p-sided antiprism is a polyhedron composed of two parallel copies of some particular p-sided polygon connected by an alternating band of the triangle. The convex polytope $R_{p}$ is obtained as a combination of a prism graph and an antiprism graph, as described in [24]. The para-line graph of $R_{p}$ is shown in Figure 1.

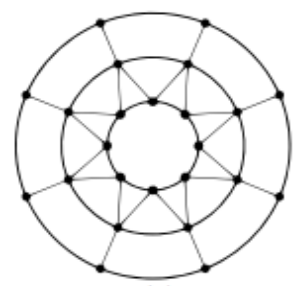

(a)

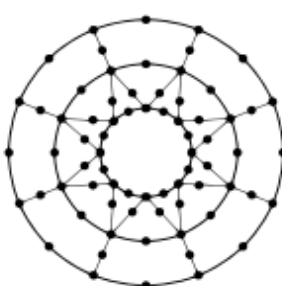

(b)

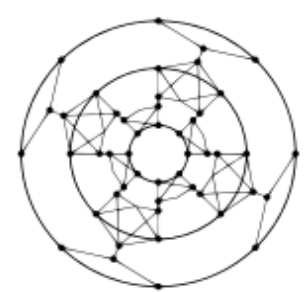

(c)

Figure 1. (a) Convex polytope $R_{8}$ (b) line graph of $R_{8}$, and (c) para-line graph of $R_{8}$.

Theorem 1. Let $G$ be the para-line graph of the convex polytope $R_{p}$. Then we have

$$
\begin{gathered}
N M(G)=2 p x^{9} y^{9}+2 p x^{9} y^{11}+p x^{11} y^{23}+2 p x^{23} y^{25}+p x^{24} y^{24}+2 p x^{25} y^{25}+2 p x^{16} y^{16} \\
+4 p x^{16} y^{17}+p x^{17} y^{17}+2 p x^{17} y^{24}+2 p x^{23} y^{24}+4 p x^{24} y^{25} .
\end{gathered}
$$

Proof. The para-line graph of the convex polytope $R_{p}$ has $25 p$ number of edges. Its edge set can be partitioned as follows:

Table 2. The edge partition of $L\left(S\left(R_{p}\right)\right)$.

\begin{tabular}{c|c|c|c}
$\left(\boldsymbol{\delta}_{\boldsymbol{u}}, \boldsymbol{\delta}_{\boldsymbol{v}}\right)$ & Cardinality & $\left(\boldsymbol{\delta}_{\boldsymbol{u}}, \boldsymbol{\delta}_{\boldsymbol{v}}\right)$ & Cardinality \\
\hline$(9,9)$ & $2 p$ & $(16,16)$ & $2 p$ \\
\hline$(9,11)$ & $2 p$ & $(16,17)$ & $4 p$ \\
\hline$(11,23)$ & $p$ & $(17,17)$ & $p$ \\
\hline$(23,25)$ & $2 p$ & $(17,24)$ & $2 p$ \\
\hline$(24,24)$ & $p$ & $(23,24)$ & $2 p$ \\
\hline$(25,25)$ & $2 p$ & $(24,25)$ & $4 p$
\end{tabular}

Now using the definition of $N M$-polynomial and Table 2, we get

$$
\begin{aligned}
& N M(G)=m_{(9,9)} x^{9} y^{9}+m_{(9,11)} x^{9} y^{11}+m_{(11,23)} x^{11} y^{23}+m_{(23,25)} x^{23} y^{25}+m_{(24,24)} x^{24} y^{24} \\
& +m_{(25,25)} x^{25} y^{25}+m_{(16,16)} x^{16} y^{16}+m_{(16,17)} x^{16} y^{17}+m_{(17,17)} x^{17} y^{17} \\
& +m_{(17,24)} x^{17} y^{24}+m_{(23,24)} x^{23} y^{24}+m_{(24,25)} x^{24} y^{25} .
\end{aligned}
$$

After putting the values of $m_{(i, j)}$ 's, the required result can be obtained easily. Hence the proof. The NM-polynomial for the para-line graph of $R_{p}$ is plotted in Figure 2.

Now using this NM-polynomial, we calculate some neighborhood degree-based topological indices of the para-line graph of the convex polytope $R_{p}$. 


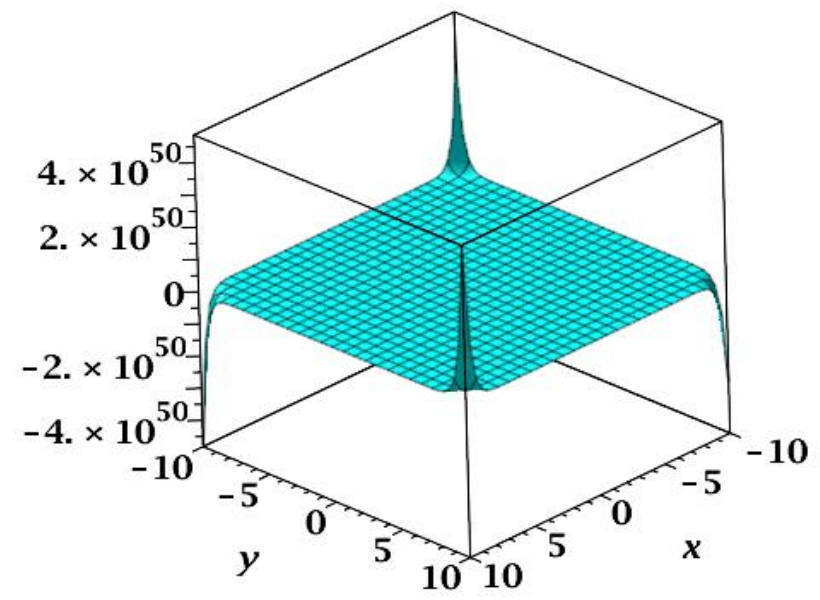

Figure 2. The $N M$-polynomial for the para-line graph of $R_{p}$ for $\mathrm{p}=2$.

Theorem 2. Let $G$ be the $L\left(S\left(R_{p}\right)\right)$. Then we have

1. $M_{1}^{\prime}(G)=956 p$,

2. $M_{2}^{*}(G)=9798 p$,

3. $F_{N}^{*}(G)=19864 p$,

4. $\quad M_{2}^{n m}(G)=0.098 p$,

5. $\quad N R_{\alpha}(G)=\left[2(9)^{2 \alpha}+2(99)^{\alpha}+(253)^{\alpha}+2(575)^{\alpha}+(576)^{\alpha}+2(625)^{\alpha}+\right.$ $\left.2(256)^{\alpha}+4(272)^{\alpha}+(289)^{\alpha}+2(408)^{\alpha}+2(552)^{\alpha}+4(600)^{\alpha}\right] p$,

6. $N D_{3}(G)=425884 p$,

7. $N D_{5}(G)=50.929 p$,

8. $\quad N H(G)=1.458 p$,

9. $\quad N I(G)=237.141 p$

10. $S(G)=30355.863 p$.

Proof. Let $N M(G)=f(x, y)=2 p x^{9} y^{9}+2 p x^{9} y^{11}+p x^{11} y^{23}+2 p x^{23} y^{25}+p x^{24} y^{24}+$ $2 p x^{25} y^{25}+2 p x^{16} y^{16}+4 p x^{16} y^{17}+p x^{17} y^{17}+2 p x^{17} y^{24}+2 p x^{23} y^{24}+4 p x^{24} y^{25}$.

Then we have

$$
\begin{aligned}
& \left(D_{x}+D_{y}\right)(f(x, y))=36 p x^{9} y^{9}+40 p x^{9} y^{11}+34 p x^{11} y^{23}+96 p x^{23} y^{25}+48 x^{24} y^{24}+ \\
& 100 p x^{25} y^{25}+64 p x^{16} y^{16}+132 p x^{16} y^{17}+34 p x^{17} y^{17}+82 p x^{17} y^{24}+94 p x^{23} y^{24}+ \\
& 196 p x^{24} y^{25} \\
& \left(D_{x} D_{y}\right)(f(x, y))=162 p x^{9} y^{9}+198 p x^{9} y^{11}+253 x^{11} y^{23}+1150 p x^{23} y^{25}+ \\
& 576 p x^{24} y^{24}+1250 p x^{25} y^{25}+512 p x^{16} y^{16}+1088 p x^{16} y^{17}+289 p x^{17} y^{17}+ \\
& 816 p x^{17} y^{24}+1104 p x^{23} y^{24}+2400 p x^{24} y^{25}, \\
& \left(D_{x}^{2}+D_{y}^{2}\right)(f(x, y))=324 p x^{9} y^{9}+404 p x^{9} y^{11}+650 p x^{11} y^{23}+2308 p x^{23} y^{25}+ \\
& 1152 p x^{24} y^{24}+2500 p x^{25} y^{25}+1024 p x^{16} y^{16}+2180 p x^{16} y^{17}+578 p x^{17} y^{17}+ \\
& 1730 p x^{17} y^{24}+2210 p x^{23} y^{24}+4804 p x^{24} y^{25},
\end{aligned}
$$




$$
\begin{aligned}
& S_{x} S_{y}(f(x, y))=\frac{2}{81} p x^{9} y^{9}+\frac{2}{99} p x^{9} y^{11}+\frac{1}{253} x^{11} y^{23}+\frac{2}{575} p x^{23} y^{25}+\frac{1}{576} p x^{24} y^{24}+ \\
& \frac{2}{625} p x^{25} y^{25}+\frac{1}{128} p x^{16} y^{16}+\frac{1}{68} p x^{16} y^{17}+\frac{1}{289} p x^{17} y^{17}+\frac{1}{204} p x^{17} y^{24}+\frac{1}{276} p x^{23} y^{24}+ \\
& \frac{1}{150} p x^{24} y^{25} \text {, } \\
& D_{x}^{\alpha} D_{y}^{\alpha}(f(x, y))=2(9)^{2 \alpha} p x^{9} y^{9}+2(99)^{\alpha} p x^{9} y^{11}+(253)^{\alpha} p x^{11} y^{23}+2(575)^{\alpha} p x^{23} y^{25}+ \\
& (576)^{\alpha} p x^{24} y^{24}+2(625)^{\alpha} p x^{25} y^{25}+2(256)^{\alpha} p x^{16} y^{16}+4(272)^{\alpha} p x^{16} y^{17}+ \\
& (289)^{\alpha} p x^{17} y^{17}+2(408)^{\alpha} p x^{17} y^{24}+2(552)^{\alpha} p x^{23} y^{24}+4(600)^{\alpha} p x^{24} y^{25} \text {, } \\
& D_{x} D_{y}\left(D_{x}+D_{y}\right)(f(x, y))=2916 p x^{9} y^{9}+3960 p x^{9} y^{11}+8602 p x^{11} y^{23}+ \\
& 55200 p x^{23} y^{25}+27648 p x^{24} y^{24}+62500 p x^{25} y^{25}+16384 p x^{16} y^{16}+35904 p x^{16} y^{17}+ \\
& 9826 p x^{17} y^{17}+33456 p x^{17} y^{24}+51888 p x^{23} y^{24}+1176000 p x^{24} y^{25} \text {, } \\
& \left(D_{x} S_{y}+S_{x} D_{y}\right)(f(x, y))=4 p x^{9} y^{9}+\frac{404}{99} p x^{9} y^{11}+\frac{650}{253} p x^{11} y^{23}+\frac{2308}{575} p x^{23} y^{25}+ \\
& 2 p x^{24} y^{24}+4 p x^{25} y^{25}+4 p x^{16} y^{16}+\frac{545}{68} p x^{16} y^{17}+\frac{578}{289} p x^{17} y^{17}+\frac{865}{204} p x^{17} y^{24}+ \\
& \frac{1105}{276} p x^{23} y^{24}+\frac{1201}{150} p x^{24} y^{25} \\
& S_{x} J(f(x, y))=p\left[\frac{1}{9} x^{18}+\frac{1}{10} x^{20}+\frac{1}{34} x^{34}+\frac{1}{24} x^{24}+\frac{1}{48} x^{48}+\frac{1}{25} x^{50}+\frac{1}{16} x^{32}+\frac{4}{33} x^{33}+\right. \\
& \left.\frac{1}{34} x^{34}+\frac{2}{41} x^{41}+\frac{2}{47} x^{47}+\frac{4}{49} x^{49}\right] \text {, } \\
& S_{x} J D_{x} D_{y}(f(x, y))=p\left[9 x^{18}+\frac{99}{10} x^{20}+\frac{253}{34} x^{34}+\frac{575}{24} x^{24}+12 x^{48}+25 x^{50}+16 x^{32}+\right. \\
& \left.\frac{1088}{33} x^{33}+\frac{17}{2} x^{34}+\frac{816}{41} x^{41}+\frac{1104}{47} x^{47}+\frac{2400}{49} x^{49}\right] \text {, } \\
& S_{x}^{3} Q_{-2} J D_{x}^{3} D_{y}^{3}(f(x, y))=p\left[259.493 x^{16}+332.75 x^{18}+494.21 x^{32}+3906.25 x^{46}+\right. \\
& 1963.333 x^{46}+4415.159 x^{48}+1242.757 x^{30}+2701.977 x^{31}+736.62 x^{32}+ \\
& \left.2289.901 x^{39}+3691.558 x^{45}+8321.855 x^{47}\right] \text {. }
\end{aligned}
$$

Using Table 1, we can easily obtain the required result.
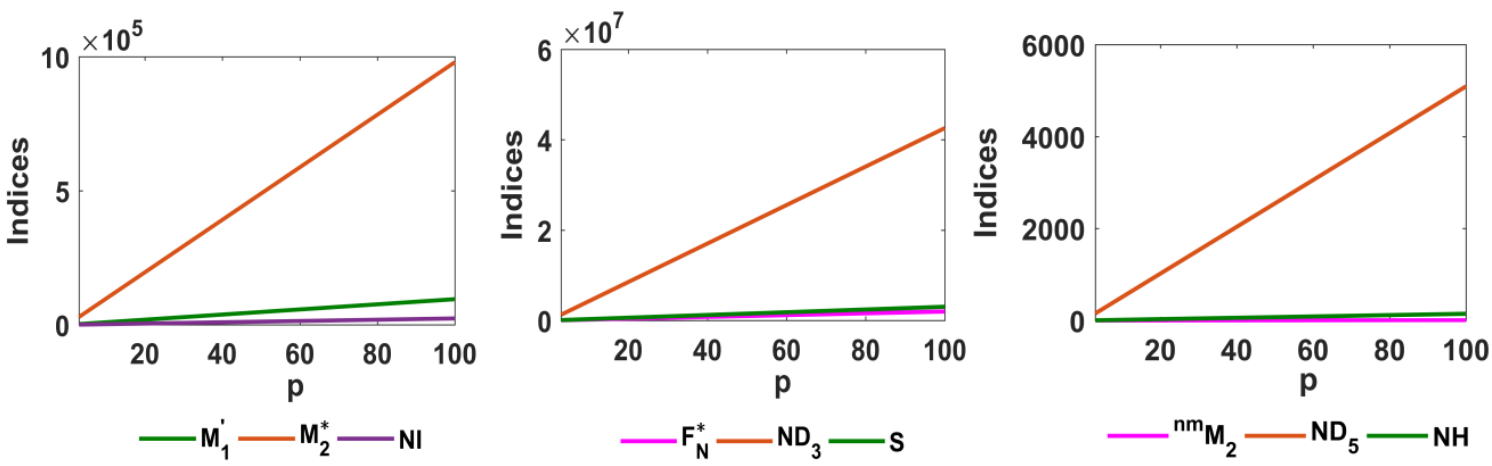

Figure 3. Graphical comparison of the indices for the para-line graph of $R_{p}$.

4.1.2. Para-line graph of the convex polytope $Q_{p}$.

The structure of convex polytope $Q_{p}$ consists of 3-sided faces, 4-sided faces, 5-sided faces, and p-sided face [24]. The para line graph of $Q_{p}$ is depicted in Figure 4. 


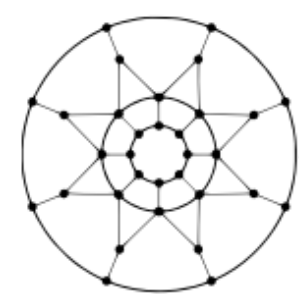

(a)

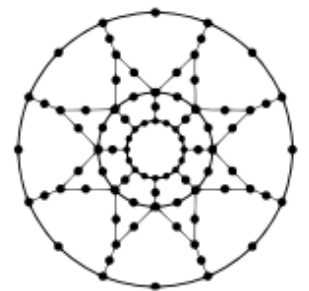

(b)

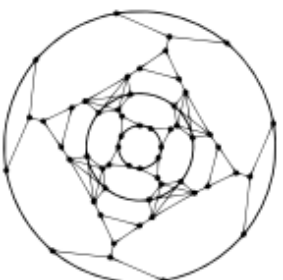

(c)

Figure 4. (a)Convex polytope $Q_{8}$ (b) line graph of $Q_{8}$, and (c) para-line graph of $Q_{8}$.

It contains $26 p$ number of edges. Its edge set can be partitioned as follow:

Table 3. The edge partition of $L\left(S\left(Q_{p}\right)\right)$.

\begin{tabular}{l|c}
$\left(\boldsymbol{\delta}_{\boldsymbol{u}}, \boldsymbol{\delta}_{\boldsymbol{v}}\right)$ & Cardinality \\
\hline$(9,9)$ & $7 p$ \\
\hline$(9,11)$ & $4 p$ \\
\hline$(11,23)$ & $p$ \\
\hline$(23,25)$ & $3 p$ \\
\hline$(24,24)$ & $11 p$
\end{tabular}

Using this edge partition, as reported in Table 3, we obtain the following theorem as previously.

Theorem 3. Let $G$ be the para-line graph of the convex polytope $Q_{p}$. Then we have $N M(G)=7 p x^{9} y^{9}+4 p x^{9} y^{11}+p x^{11} y^{11}+3 p x^{11} y^{13}+11 p x^{23} y^{23}$.

The NM-polynomial for the para-line graph of $Q_{p}$ is plotted in Figure 5.

Applying Theorem 3 and Table 1, we compute the neighborhood degree-based indices for $L\left(S\left(Q_{p}\right)\right)$ in the following theorem.

Theorem 4. Let $G$ be the $L\left(S\left(Q_{p}\right)\right)$. Then we have

1. $M_{1}^{\prime}(G)=806 p$,

2. $M_{2}^{*}(G)=7332 p$,

3. $F_{N}^{*}(G)=14692 p$,

4. $M_{2}^{n m}(G)=0.167 p$,

5. $N R_{\alpha}(G)=\left[7(9)^{2 \alpha}+4(99)^{\alpha}+(121)^{\alpha}+3(143)^{\alpha}+11(529)^{\alpha}\right] p$,

6. $N D_{3}(G)=298758 p$,

7. $N D_{5}(G)=52.246 p$,

8. $N H(G)=1.997 p$,

9. $N I(G)=201.175 p$,

10. $S(G)=21735.249 p$.

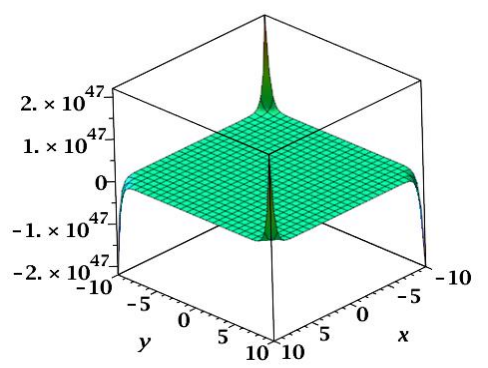

Figure 5.The $N M$-polynomial for the para-line graph of $Q_{p}$ for $\mathrm{p}=2$. 

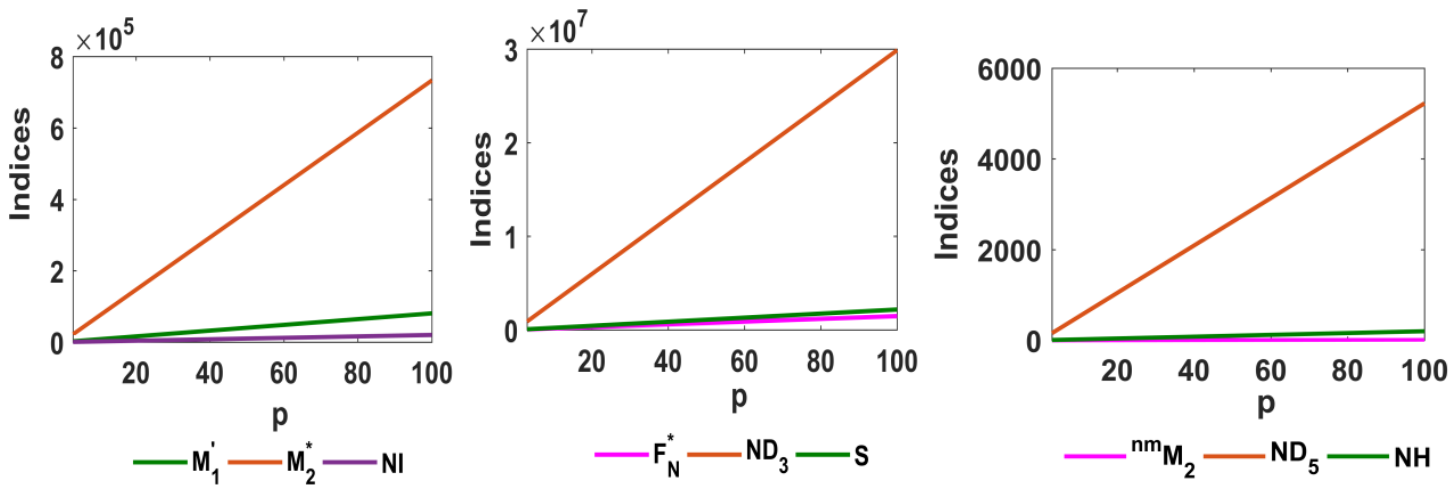

Figure 6. Graphical comparison of the indices for the para-line graph of $Q_{p}$.

4.1.3. Para-line graph of the convex polytope $\mathrm{D}_{\mathrm{p}}$.

The structure of convex polytope $D_{p}$ consists of 5-sided faces and p-sided face as defined in [23]. The para-line graph of $D_{p}$ is shown in Figure 7.

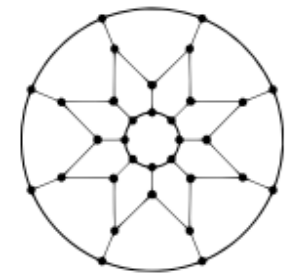

(a)

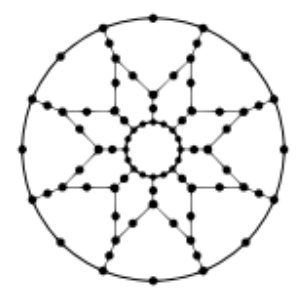

(b)

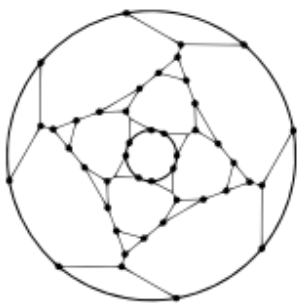

(c)

Figure 7. (a) Convex polytope $D_{8}$ (b) line graph of $D_{8}$, and (c) para-line graph of $D_{8}$.

The para-line graph of $D_{p}$ has $18 \mathrm{p}$ edges, all of which has end vertices with neighborhood degree $\left(\delta_{u}, \delta_{v}\right)=(9,9)$. The $N M$ polynomial of $D_{p}$ is obtained in the following theorem.

Theorem 5. Let $G$ be the para-line graph of the convex polytope $D_{p}$. Then we have $N M(G)=18 p x^{9} y^{9}$.

The NM-polynomial for the para-line graph of $D_{p}$ is plotted in Figure 8.

From Theorem 5, the neighborhood degree-based topological indices for the para-line graph of the convex polytope $D_{p}$ are recovered in the following theorem.

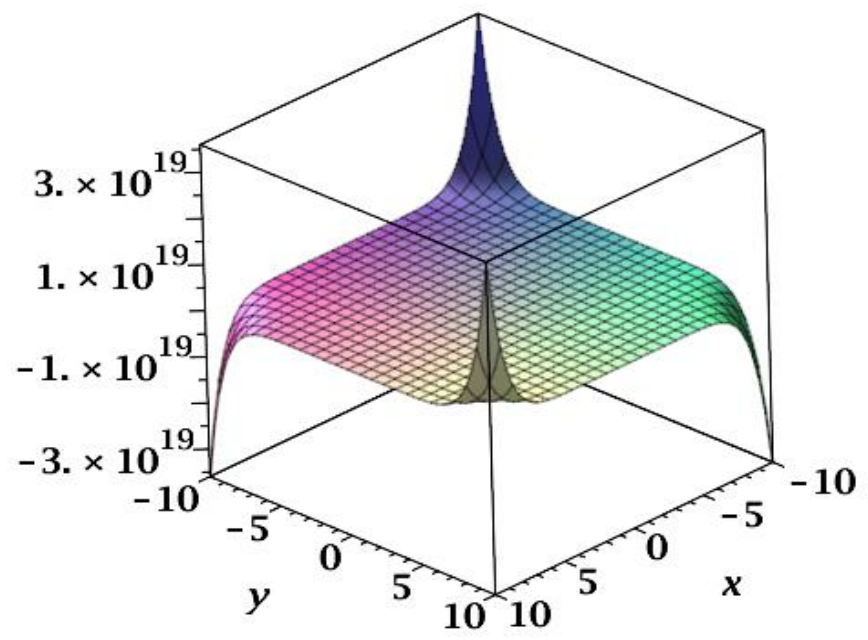

Figure 8. The $N M$-polynomial for the para-line graph of $D_{p}$ for $\mathrm{p}=2$. 
Theorem 6. Let $G$ be the $L\left(S\left(D_{p}\right)\right)$. Then we have

1. $M_{1}^{\prime}(G)=324 p$,

2. $M_{2}^{*}(G)=1458 p$,

3. $F_{N}^{*}(G)=2916 p$,

4. $\quad M_{2}^{n m}(G)=0.222 p$,

5. $\quad N R_{\alpha}(G)=18(9)^{2 \alpha} p$,

6. $N D_{3}(G)=26244 p$

7. $N D_{5}(G)=36 p$,

8. $\quad N H(G)=2 p$,

9. $\quad N I(G)=81 p$,

10. $S(G)=2335.434 p$.
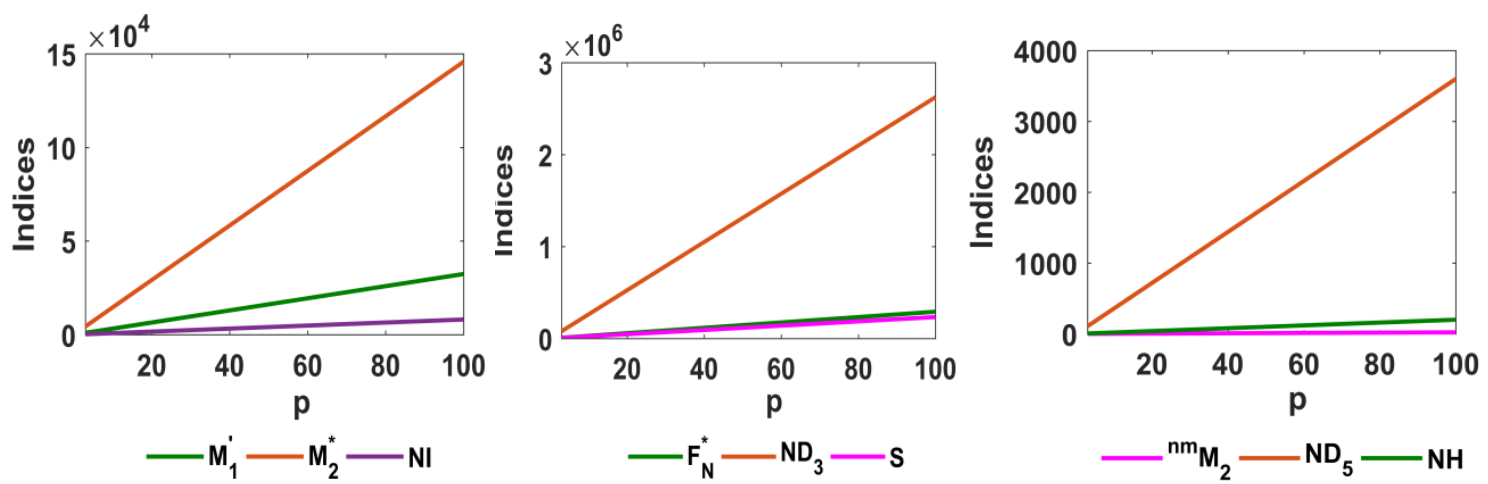

Figure 9. Graphical comparison of the indices for the para-line graph of $D_{p}$.

\subsection{Comparative study.}

In this section, our goal is to create a numerical and graphical comparison of different indices for the considered structures. We construct this study in two parts: firstly, all the indices taking three at a time are considered for three different structures (see Figures 3, 6, 9) and then the three para-line graphs are taken into account in one frame for different indices (see Figures $10,11,12,13)$. Numerical values of the indices for $p=3,4, \ldots, 10$, are listed in Tables 4-6, and their corresponding graphical representations are shown in Figures 10-12.

We consider $G_{1}, G_{2}$ and $G_{2}$ to represent $L\left(S\left(R_{p}\right)\right), L\left(S\left(Q_{p}\right)\right)$ and $L\left(S\left(D_{p}\right)\right)$, respectively, in Tables 4-6 and in the current discussion. In each figure, the graph parameters are considered along the horizontal axis, and the indices are along the vertical axis. Graphical representations of all indices for $G_{1}$ are shown in Figure 3. The indices for $G_{2}$ are plotted in Figure 6. The indices for $G_{3}$ are plotted in Figure 9. The third version of the Zagreb index, the neighborhood second Zagreb index, and the neighborhood forgotten topological index are listed in Table 4, and they are compared graphically in Figure 10. The neighborhood second modified Zagreb index, the third and fifth NDe indices are computed in Table 5, and the values are depicted in Figure 11. The neighborhood harmonic index, the neighborhood inverse sum index, and the Sanskriti index are computed in Table 6, and the results are compared graphically in Figure 12. The neighborhood general Randic index is plotted for a certain range of $p, \alpha$ in Figure 13, where the logarithm scale is used along the vertical axis to express the results fairly. It is evident from different Tables and Figures that the indices increase as the parameters of the graph increase. From figures, some comments can be drawn. 


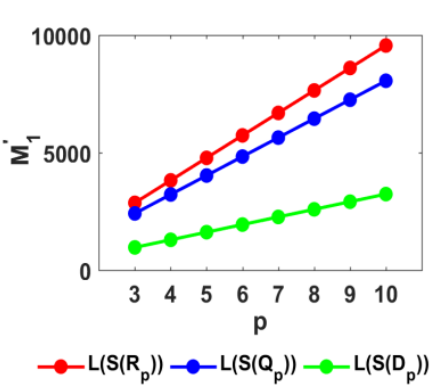

(a)

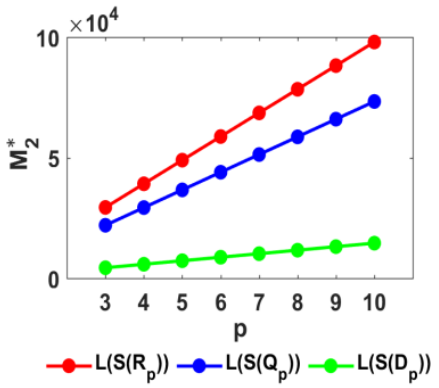

(b)

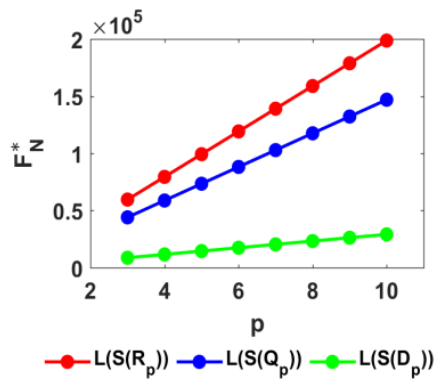

(c)

Figure 10. Graphical comparison of $M_{1}^{\prime}, M_{2}^{*}$ and $F_{N}^{*}$ for the structures under consideration.

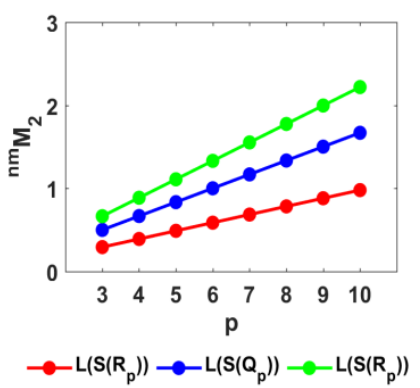

(a)

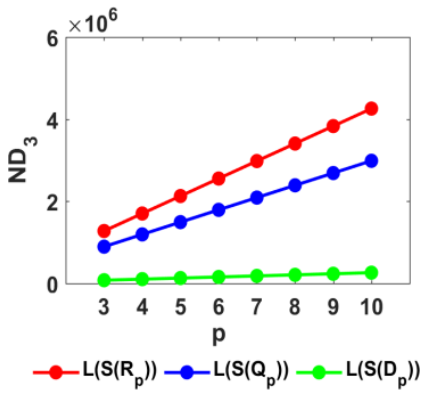

(b)

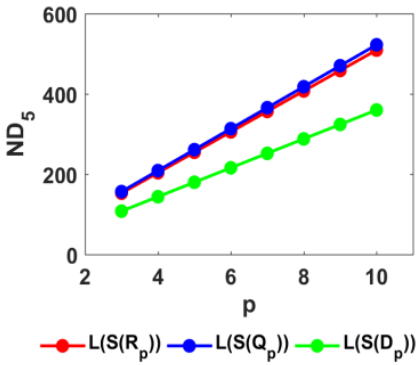

(c)

Figure 11. Graphical comparison of $M_{2}^{n m}, N D_{3}$ and $N D_{5}$ for the structures under consideration.

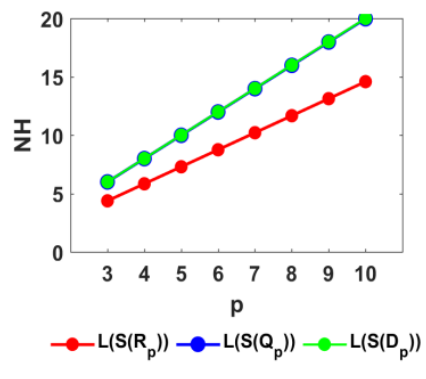

(a)

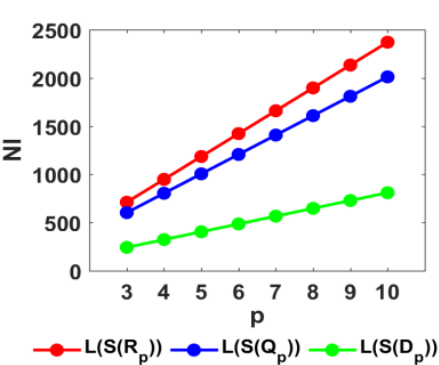

(b)

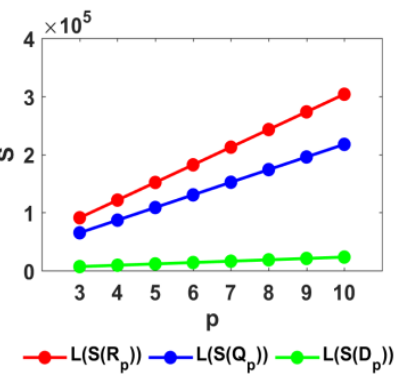

(c)

Figure 12. Graphical comparison of $N H, N I$, and $S$ for the structures under consideration.

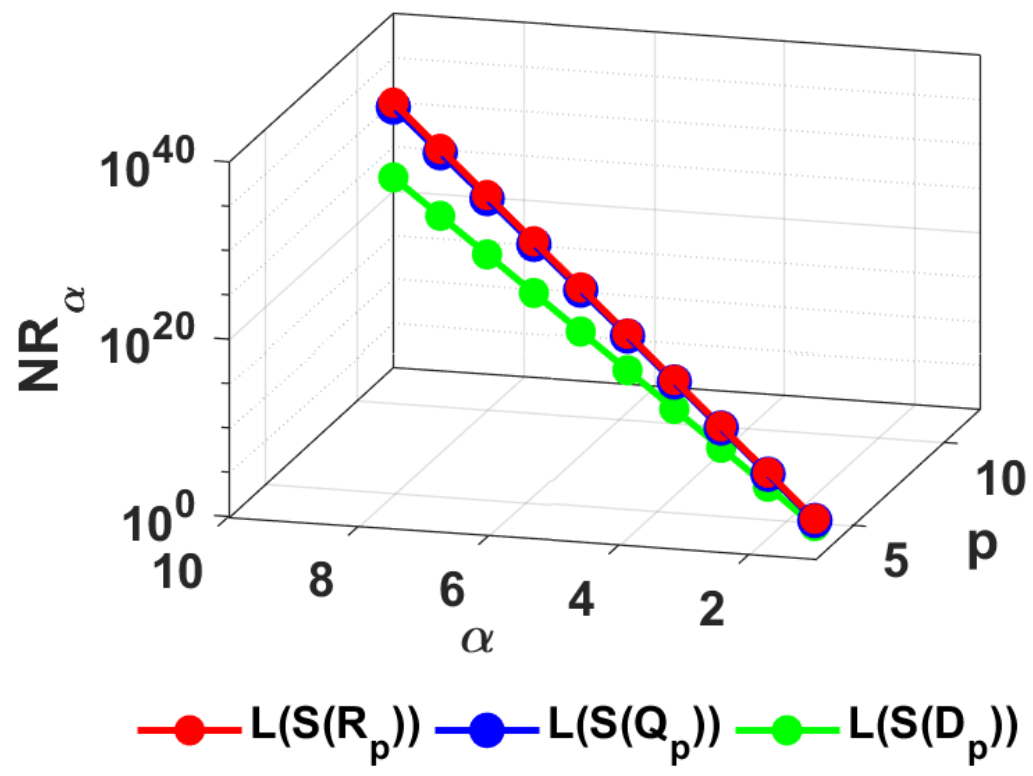

Figure 13. Graphical comparison of $N R_{\alpha}$ for the structures under consideration. 
Table 4. Numerical values of $M_{1}^{\prime}, M_{2}^{*}$ and $F_{N}^{*}$ for the three para-line graphs.

\begin{tabular}{l|l|l|l|l|l|l|l|l|l}
$\mathbf{p}$ & $\boldsymbol{M}_{\mathbf{1}}^{\prime}\left(\boldsymbol{G}_{\mathbf{1}}\right)$ & $\boldsymbol{M}_{\mathbf{1}}^{\prime}\left(\boldsymbol{G}_{\mathbf{2}}\right)$ & $\boldsymbol{M}_{\mathbf{1}}^{\prime}\left(\boldsymbol{G}_{\mathbf{3}}\right)$ & $\boldsymbol{M}_{\mathbf{2}}^{*}\left(\boldsymbol{G}_{\mathbf{1}}\right)$ & $\boldsymbol{M}_{\mathbf{2}}^{*}\left(\boldsymbol{G}_{\mathbf{2}}\right)$ & $\boldsymbol{M}_{\mathbf{2}}^{*}\left(\boldsymbol{G}_{\mathbf{3}}\right)$ & $\boldsymbol{F}_{\boldsymbol{N}}^{*}\left(\boldsymbol{G}_{\mathbf{1}}\right)$ & $\boldsymbol{F}_{\boldsymbol{N}}^{*}\left(\boldsymbol{G}_{\mathbf{2}}\right)$ & $\boldsymbol{F}_{\boldsymbol{N}}^{*}\left(\boldsymbol{G}_{\mathbf{3}}\right)$ \\
\hline 3 & 2868 & 2418 & 972 & 29394 & 21996 & 4374 & 59592 & 44076 & 8748 \\
\hline 4 & 3824 & 3224 & 1296 & 39192 & 29328 & 5832 & 79456 & 58768 & 11664 \\
\hline 5 & 4780 & 4030 & 1620 & 48990 & 36660 & 7290 & 99320 & 73460 & 14580 \\
\hline 6 & 5736 & 4836 & 1944 & 58788 & 43992 & 8748 & 119184 & 88152 & 17496 \\
\hline 7 & 6692 & 5642 & 2268 & 68586 & 51324 & 10206 & 139048 & 102844 & 20412 \\
\hline 8 & 7648 & 6448 & 2592 & 78384 & 58656 & 11664 & 158912 & 117536 & 23328 \\
\hline 9 & 8604 & 7254 & 2916 & 88182 & 65988 & 13122 & 178776 & 132228 & 26244 \\
\hline 10 & 9560 & 8060 & 3240 & 97980 & 73320 & 14580 & 198640 & 146920 & 29160
\end{tabular}

Table 5. Numerical values of $M_{2}^{n m}, N D_{3}$ and $N D_{5}$ for the three para-line graphs.

\begin{tabular}{l|l|l|l|l|l|l|l|l|l}
$\mathbf{p}$ & $\boldsymbol{M}_{\mathbf{2}}^{\text {nm }}\left(\boldsymbol{G}_{\mathbf{1}}\right)$ & $\boldsymbol{M}_{\mathbf{2}}^{\text {nm }}\left(\boldsymbol{G}_{\mathbf{2}}\right)$ & $\boldsymbol{M}_{\mathbf{2}}^{\text {nm }}\left(\boldsymbol{G}_{\mathbf{3}}\right)$ & $\boldsymbol{N D}_{\mathbf{3}}\left(\boldsymbol{G}_{\mathbf{1}}\right)$ & $\boldsymbol{N D}_{\mathbf{3}}\left(\boldsymbol{G}_{\mathbf{2}}\right)$ & $\boldsymbol{N D}_{\mathbf{3}}\left(\boldsymbol{G}_{\mathbf{3}}\right)$ & $\boldsymbol{N D}_{\mathbf{5}}\left(\boldsymbol{G}_{\mathbf{1}}\right)$ & $\boldsymbol{N D}_{\mathbf{5}}\left(\boldsymbol{G}_{\mathbf{2}}\right)$ & $\boldsymbol{N D}_{\mathbf{5}}\left(\boldsymbol{G}_{\mathbf{3}}\right)$ \\
\hline 3 & 0.294 & 0.501 & 0.666 & 1277652 & 896274 & 78732 & 152.787 & 156.738 & 108 \\
\hline 4 & 0.392 & 0.668 & 0.888 & 1703536 & 1195032 & 104976 & 203.716 & 208.984 & 144 \\
\hline 5 & 0.49 & 0.835 & 1.11 & 2129420 & 1493790 & 131220 & 254.645 & 261.23 & 180 \\
\hline 6 & 0.588 & 1.002 & 1.332 & 2555304 & 1792548 & 157464 & 305.574 & 313.476 & 216 \\
\hline 7 & 0.686 & 1.169 & 1.554 & 2981188 & 2091306 & 183708 & 356.503 & 365.722 & 252 \\
\hline 8 & 0.784 & 1.336 & 1.776 & 3407072 & 2390064 & 209952 & 407.432 & 417.968 & 288 \\
\hline 9 & 0.882 & 1.503 & 1.998 & 3832956 & 2688822 & 236196 & 458.361 & 470.214 & 324 \\
\hline 10 & 0.98 & 1.67 & 2.22 & 4258840 & 2987580 & 262440 & 509.29 & 522.46 & 360
\end{tabular}

Table 6. Numerical values of $N H, N I$ and $S$ for the three para-line graphs.

\begin{tabular}{l|l|l|l|l|l|l|l|l|l}
$\mathbf{p}$ & $\boldsymbol{N H}\left(\boldsymbol{G}_{\mathbf{1}}\right)$ & $\mathbf{N H}\left(\boldsymbol{G}_{\mathbf{2}}\right)$ & $\boldsymbol{N H}\left(\boldsymbol{G}_{\mathbf{3}}\right)$ & $\boldsymbol{N} \boldsymbol{I}\left(\boldsymbol{G}_{\mathbf{1}}\right)$ & $\boldsymbol{N} \boldsymbol{I}\left(\boldsymbol{G}_{\mathbf{2}}\right)$ & $\boldsymbol{N I}\left(\boldsymbol{G}_{\mathbf{3}}\right)$ & \multicolumn{1}{|c}{$\boldsymbol{S}\left(\boldsymbol{G}_{\mathbf{1}}\right)$} & $\boldsymbol{S}\left(\boldsymbol{G}_{\mathbf{2}}\right)$ & $\boldsymbol{S}\left(\boldsymbol{G}_{\mathbf{3}}\right)$ \\
\hline 3 & 4.374 & 5.991 & 6 & 711.423 & 603.525 & 243 & 91067.589 & 65205.747 & 7006.302 \\
\hline 4 & 5.832 & 7.988 & 8 & 948.564 & 804.7 & 324 & 121423.452 & 86940.996 & 9341.736 \\
\hline 5 & 7.29 & 9.985 & 10 & 1185.705 & 1005.875 & 405 & 151779.315 & 108676.245 & 11677.17 \\
\hline 6 & 8.748 & 11.982 & 12 & 1422.846 & 1207.05 & 486 & 182135.178 & 130411.494 & 14012.604 \\
\hline 7 & 10.206 & 13.979 & 14 & 1659.987 & 1408.225 & 567 & 212491.041 & 152146.743 & 16348.038 \\
\hline 8 & 11.664 & 15.976 & 16 & 1897.128 & 1609.4 & 648 & 242846.904 & 173881.992 & 18683.472 \\
\hline 9 & 13.122 & 17.973 & 18 & 2134.269 & 1810.575 & 729 & 273202.767 & 195617.241 & 21018.906 \\
\hline 10 & 14.58 & 19.97 & 20 & 2371.41 & 2011.75 & 810 & 303558.63 & 217352.49 & 23354.34
\end{tabular}

In comparison with other indices, the third NDe index has the most dominating nature, whereas the neighborhood second modified Zagreb index grows slowly. The behavior of neighborhood forgotten topological index and Sanskruti index are very closed. From the vertical axis, it seems that the indices for different structures grow up in the following order: $I\left(G_{3}\right)<I\left(G_{2}\right)<I\left(G_{1}\right)$, except $M_{2}^{n m}, N D_{5}$ and $N H$. From Figure 11(a), it is obvious that $M_{2}^{n m}\left(G_{1}\right)<M_{2}^{n m}\left(G_{2}\right)<M_{2}^{n m}\left(G_{3}\right)$. Figure $11(\mathrm{c})$ reveals that $N D_{5}\left(G_{3}\right)<N D_{5}\left(G_{1}\right)<$ $N D_{5}\left(G_{2}\right)$. However $N D_{5}\left(G_{2}\right)$ is very close to $N D_{5}\left(G_{1}\right)$. One can found from Figure 12(a) that $\mathrm{NH}\left(G_{2}\right)$ and $N H\left(G_{3}\right)$ are dominating $N H\left(G_{1}\right)$, but it is difficult to distinguish them as their graphs are overlapped. They are very closed. When we look into Table 6 , it is evident that $N H\left(G_{2}\right)<N H\left(G_{3}\right)$. For each structure, indices have the following order: $M_{2}^{n m}<N H<$ $N D_{5}<N I<M_{1}^{\prime}<M_{2}^{*}<F_{N}^{*}<S<N D_{3}$. Thus, we can state that all the indices behave differently in each structure discussed above.

\section{Conclusions}

In this work, we have considered three types of convex polytopes. Firstly we have obtained NM-polynomial of the paraline graph of the convex polytopes. Later some neighborhood degree sum based topological indices including the third version of Zagreb index, neighborhood second Zagreb index, the neighborhood was forgotten topological index, neighborhood second modified Zagreb index, neighborhood general Randic index, third NDe index, fifth NDe index, neighborhood harmonic index, neighborhood inverse sum index, and Sanskruti index for the structures under consideration had been derived using NM-polynomial. 
The results are interpreted and compared graphically. This work is helpful in understanding the topology of the aforesaid structures.

\section{Funding}

This research received no external funding.

\section{Acknowledgments}

The first author is very obliged to the Department of Science and Technology (DST), Government of India, for the Inspire Fellowship [IF170148].

\section{Conflicts of Interest}

The authors declare no conflict of interest.

\section{References}

1. Wiener, H. Structural determination of the paraffin boiling points. J. Am. Chem. Soc. 1947, 69, 17-20, https://doi.org/10.1021/ja01193a005.

2. Hosamani, S.M. Computing Sanskruti index of certain nanostructures. J. Appl. Math. Comput. 2017, 54, 425-433, https://doi.org/10.1007/s12190-016-1016-9.

3. Ghorbani, M.; Hosseinzadeh, M.A. The third version of Zagreb index. Discrete Math. Algorithm Appl. 2013, 5, https://doi.org/10.1142/S1793830913500390.

4. Kulli, V.R. Neighborhood Indices of Nanostructures. International Journal of Current Research in Science and Technology 2019, 5, 1-14.

5. Kulli, V. Multiplicative Neighborhood Indices. Annals of Pure and Applied Mathematics 2019, 19, 175-181, https://doi.org/10.22457/apam.614v19n2a6

6. Mondal, S.; De, N.; Pal, A. On Neighbourhood Zagreb index of product graphs. arXiv:1805.052732018.

7. Mondal, S.; De, N.; Pal, A. On some new neighbourhood degree based indices. Acta Chemica Iasi 2019, 27, 31-46, https://doi.org/10.2478/achi-2019-0003.

8. Mondal, S.; De, N.; Pal, A. QSPR analysis of some novel neighborhood degree based topological descriptors. arXiv:1906.066602019.

9. Gutman, I. Some properties of the Wiener polynomials. Graph Theory Notes N.Y. 1993, 125, 13-18.

10. Alamian, V.; Bahrami, A.; Edalatzadeh, B. PI Polynomial of V-Phenylenic nanotubes and nanotori. Int. J. Mole. Sci. 2008, 9, 229-234, https://doi.org/10.3390/ijms9030229

11. Farahani, M. R. Computing theta polynomial, and theta index of V-phenylenic planar, nanotubes and nanotoris. Int. J. Theoretical Chem.2013, 1, 01-09.

12. Ashrafi, A.R.; Manoochehrian, B.; Azari, H.Y. On the PI polynomial of a graph. Util. Math. 2006, 71, 97108.

13. Shao, Z.; Siddiqui, M. K.; Muhammad, M.H. Computing Zagreb Indices and Zagreb Polynomials for Symmetrical Nanotubes. Symmetry 2018, 10,244-259, https://doi.org/10.3390/sym10070244.

14. Deutsch, E.; Klavzar, S. M-Polynomial, and degree-based topological indices. Iran. J. Math. Chem. 2015, 6, 93-102, https://doi.org/10.22052/IJMC.2015.10106.

15. Kwun, Y.C.; Munir, M.; Nazeer, W.; Rafque, S. Kang, S.M. M-Polynomials and topological indices of VPhenylenic Nanotubes and Nanotori. Sci. Rep. 2017, 7, https://doi.org/10.1038/s41598-017-08309-y.

16. Mondal, S.; De, N.; Pal, A. The M-Polynomial of Line graph of Subdivision graphs. Commun. Fac. Sci. Univ. Ank. Ser. Al Math. Stat. 2019, 68, 2104-2116, https://doi.org/10.31801/cfsuasmas.587655.

17. Mondal, S.; Siddiqui, M.K.; De, N.; Pal, A. Neighborhood M-polynomial of crystallographic structures. Biointerface Res. Appl. Chem. 2021, 11, 9372-9381, https://doi.org/10.33263/BRIAC112.93729381.

18. Ranjini, P.S.; Lokesha, V.; Ranjan, M.A. On Zagreb indices of the subdivision graphs. Int. J. Math. Sci. Eng. Appl. 2010, 4, 221-228.

19. Gutman, I.; Estrada, E. Topological indices based on the line graph of the molecular graph.J. Chem. Inf. Comp. Sci. 1996, 36, 541-543, https://doi.org/10.1021/ci950143i

20. Asif, F.; Zahid, Z.; Zafar, S.; Farahani, M.R.; Gao, W. On topological properties of some convex polytopes by using line operator on their subdivisions. Hacet. J. Math. Stat. 2019, 49, 1-10, https://doi.org/10.15672/HJMS.2019.671

21. Foruzanfar, Z.; Asif, F.; Zahid, Z.; Zafar, S.; Farahani, M.R. $A B C_{4}$ and $G A_{5}$ indices of para-line graph of some convex polytopes. Stat., Optim. Inf. Comput. 2019, 7, 192-197, https://doi.org/10.19139/soic.v7i1.346.

22. Grünbaum, B. Graduate text in mathematics convex polytopes. Springer-Verlag, New York, 2003. 
23. Baca, M. Labellings of two classes of convex polytopes. Util. Math.1988, 34, 24-31.

24. Baca, M. On magic labellings of convex polytopes.Ann. Discrete Math. 1992, 51, 13-16, https://doi.org/10.1016/S0167-5060(08)70599-5.

25. Liu, J.B.; Zhao, J.; He, H.; Shao, Z. Valency-Based Topological Descriptors and Structural Property of the Generalized Sierpinski Networks. J. Stat. Phys. 2019, 177, 1131-1147, https://doi.org/10.1007/s10955-01902412-2.

26. Mondal, S.; De, N.; Pal, A. On some general neighborhood degree based topological indices. Int. J. Appl. Math. 2019, 32, 1037-1049, https://doi.org/10.1142/S2661335219500060

27. Liu, J.B.; Zhao, J.; Min, J.; Cao, J.D. On the Hosoya index of graphs formed by a fractal graph. Fractals 2019, 27, https://doi.org/10.1142/S0218348X19501354.

28. Mondal, S.; De, N.; Pal, A. Multiplicative Degree Based Topological Indices of Nanostar Dendrimer. Biointerface Res. Appl. Chem. 2021, 11, 7700-7711, https://doi.org/10.33263/BRIAC111.77007711 .

29. Mondal, S.; De, N.; Pal, A. Topological properties of Graphene using some novelneighborhood degree-based topological indices. Int. J. Ind. Math. 2020, 11, https://doi.org/10.1142/S2661335219500060

30. Liu, J.B.; Zhao, J.; Cai, Z. On the generalized adjacency, Laplacian and signless Laplacian spectra of the weighted edge corona networks. Physica A. 2020, 540, 123-133, https://doi.org/10.1016/j.physa.2019.123073. 